\title{
Searching for production of dark matter in association with top quarks at the LHC
}

\author{
Ulrich Haisch $^{a}$ and Giacomo Polesello ${ }^{b}$ \\ ${ }^{a}$ Max Planck Institute for Physics, \\ Föhringer Ring 6, 80805 München, Germany \\ ${ }^{b}$ INFN, Sezione di Pavia, \\ Via Bassi 6, 27100 Pavia, Italy \\ E-mail: haisch@mpp.mpg.de, giacomo.polesello@cern.ch
}

ABSTRACT: In the framework of spin-0 s-channel dark matter (DM) simplified models, we reassess the sensitivity of future LHC runs to the production of DM in association with top quarks. We consider two different missing transverse energy $\left(E_{T}^{\text {miss }}\right)$ signatures, namely production of DM in association with either a $t \bar{t}$ pair or a top quark and a $W$ boson, where the latter channel has not been the focus of a dedicated analysis prior to this work. Final states with two leptons are studied and a realistic analysis strategy is developed that simultaneously takes into account both channels. Compared to other existing search strategies the proposed combination of $t \bar{t}+E_{T}^{\text {miss }}$ and $t W+E_{T}^{\text {miss }}$ production provides a significantly improved coverage of the parameter space of spin- 0 s-channel DM simplified models.

KEYwords: Dark matter, Hadron-Hadron scattering (experiments)

ARXIV EPRINT: 1812.00694 


\section{Contents}

1 Introduction $\quad 1$

2 Preliminaries 2

3 Unitarity considerations 3

4 Angular correlations of the $t W+E_{T}^{\text {miss }}$ signal $\quad 5$

$5 \quad$ MC generation and detector simulation $\quad 8$

$6 \quad$ Analysis strategy 9

$\begin{array}{lll}7 & \text { Results } & 14\end{array}$

8 Conclusions $\quad 15$

\section{Introduction}

In models where dark matter $(\mathrm{DM})$ is pair-produced via an $s$-channel process (see e.g. [13]) the corresponding mediators have to be singlets under both the QCD and QED part of the standard model (SM) gauge group. Gauge invariance then implies that pure vector or axialvector mediators have to couple flavour universally to the SM quarks, an option that is severely constrained by the existing LHC searches for dijet and/or dilepton resonances. The couplings of scalar and pseudoscalars mediators to quarks are instead restricted by the stringent bounds on flavour changing neutral current processes. The simplest way to avoid the latter limits consists in invoking the minimal flavour violation (MFV) hypothesis [4]. In the spin-0 case, this hypothesis implies that the couplings of the mediators to SM matter are proportional to the fermion masses. One is thus naturally led to consider scalar and pseudoscalar mediators that couple most strongly to the third generation. Similar to the SM Higgs boson, such states can be produced at the LHC through loop-induced gluon fusion or in association with top (or bottom) quarks before they decay to the heaviest kinematically allowed SM final state or DM. Drell-Yan production instead provides generically only very weak constraints on MFV spin-0 s-channel DM simplified models.

In this work, we reassess the sensitivity of future LHC runs to the production of DM in association with top quarks in the framework of spin-0 s-channel DM simplified models. We focus on missing transverse energy $\left(E_{T}^{\text {miss }}\right)$ signatures with two leptons in the final state, and thus consider the two channels $t \bar{t}+E_{T}^{\text {miss }}$ and $t W+E_{T}^{\text {miss }}$. While the former process has received quite some attention (see [5-10] for recent theoretical studies), singletop processes involving significant amounts of $E_{T}^{\text {miss }}$ have been considered only by a few 
authors. In the publication [11] it has first been pointed out that production of DM in association with a single top can be used to increase the reach of $t \bar{t}+E_{T}^{\text {miss }}$ analyses (see very recently also $[12,13])$. The work [14] has then shown that a dedicated search for $t$-channel $t j+E_{T}^{\text {miss }}$ production can provide an interesting stand-alone DM signal at the LHC. Both of these studies have been performed in the context of spin-0 $s$-channel DM simplified models. The article [15] has finally studied single-top plus $E_{T}^{\text {miss }}$ production in an ultraviolet (UV) complete model with two Higgs doublets (2HDM) and an extra pseudoscalar mediator, known as the $2 \mathrm{HDM}+\mathrm{a}$ model [16-21]. In the framework of the $2 \mathrm{HDM}+\mathrm{a}$ model it has been shown that $t W+E_{T}^{\text {miss }}$ is the most interesting channel because it can be resonantly procedure via the exchange of a charged Higgs boson. A dedicated analysis of the $t W+E_{T}^{\text {miss }}$ final state in spin-0 $s$-channel DM simplified models has instead not been performed so far. The main goal of this work is to close this gap by developing an analysis which provides an improved coverage of the parameter space of spin- $0 s$-channel DM simplified models via a combined two-lepton search for $t \bar{t}+E_{T}^{\text {miss }}$ and $t W+E_{T}^{\text {miss }}$ production.

The outline of this article is as follows. In section 2 we describe the structure of the DM simplified models that we consider. Section 3 contains a discussion of unitarity violation in DM plus top-quark production. From this discussion one can conclude that calculations of the $t W+E_{T}^{\text {miss }}$ signature in the context of spin-0 $s$-channel DM simplified models lead to meaningful results at LHC energies. The angular correlations of the $t W+E_{T}^{\text {miss }}$ signal are discussed in section 4, while a brief description of our Monte Carlo (MC) and detector simulations is presented in section 5. Our actual analysis strategy can be found in section 6 . It spells out all selection criteria and illustrates their impact on the DM signals and the SM background. In section 7, we present the numerical results of our analysis, providing a detailed evaluation of the achievable sensitivity of $t \bar{t}+E_{T}^{\text {miss }}$ and $t W+E_{T}^{\text {miss }}$ production at future LHC runs. Constraints on the parameter space of the spin- $0 s$-channel DM simplified models are also derived in this section. We conclude in section 8.

\section{Preliminaries}

The spin-0 $s$-channel DM simplified models that we are considering in our article can be described by the following interactions (see for instance [1-3])

$$
\begin{aligned}
& \mathcal{L}_{\phi} \supset-g_{\chi} \phi \bar{\chi} \chi-\frac{\phi}{\sqrt{2}} \sum_{q=u, d, s, c, b, t} g_{q} y_{q} \bar{q} q, \\
& \mathcal{L}_{a} \supset-i g_{\chi} a \bar{\chi} \gamma_{5} \chi-i \frac{a}{\sqrt{2}} \sum_{q=u, d, s, c, b, t} g_{q} y_{q} \bar{q} \gamma_{5} q .
\end{aligned}
$$

Here $\phi(a)$ is a scalar (pseudoscalar), $\chi$ represents the DM particle assumed to be a Dirac fermion, $g_{\chi}$ is a dark-sector Yukawa coupling, $y_{q}=\sqrt{2} m_{q} / v$ are the SM quark Yukawa couplings with $m_{q}$ the mass of the relevant quark $q$ and $v \simeq 246 \mathrm{GeV}$ the Higgs vacuum expectation value, and $\gamma_{5}$ finally denotes the fifth Dirac matrix.

The Lagrangians $\mathcal{L}_{\phi / a}$ are not invariant under the SM gauge group, meaning that additional particles and/or interactions need to be invoked to embed (2.1) into a gaugeinvariant structure at the electroweak scale. Scalar and pseudoscalar couplings of the 

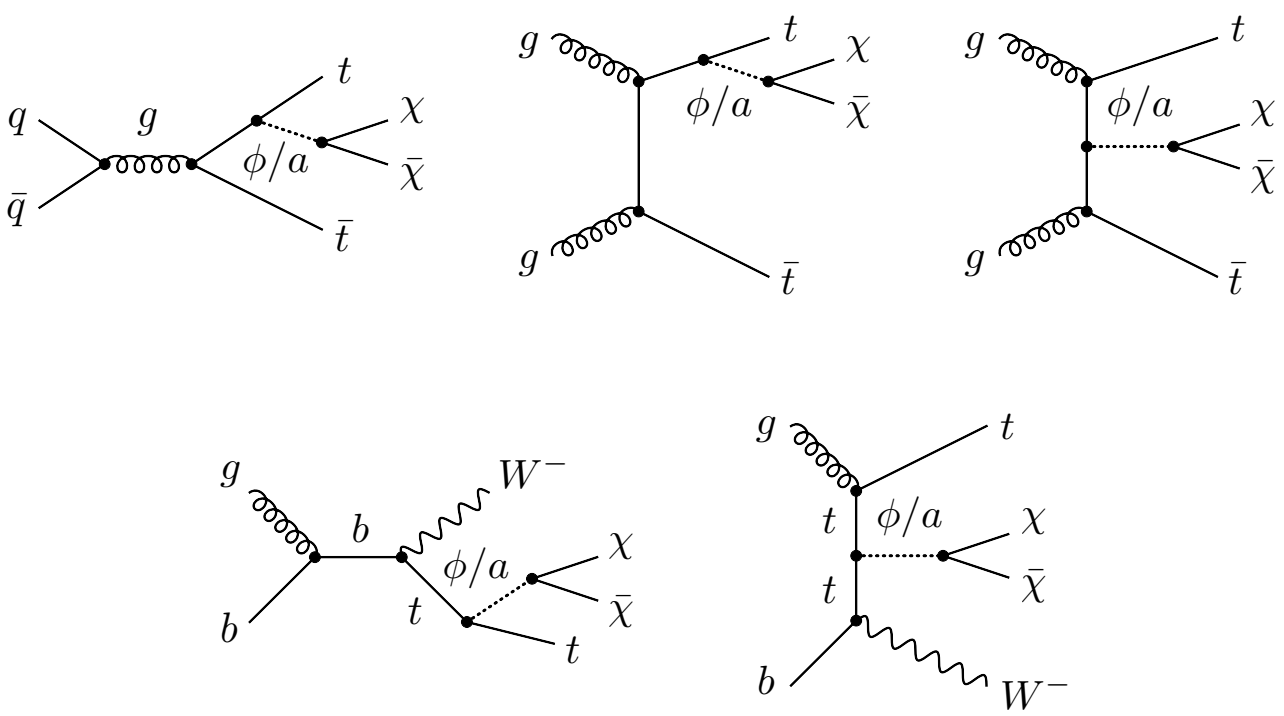

Figure 1. Representative Feynman diagrams for $t \bar{t}+E_{T}^{\text {miss }}$ (upper row) and $t W+E_{T}^{\text {miss }}$ (lower row) production that involve the exchange of a scalar $(\phi)$ or pseudoscalar $(a)$ mediator with the interactions specified in (2.1).

form (2.1) do for example arise in 2HDM extensions with an extra spin-0 gauge singlet [1622]. In fact, in models of this type there is a well-defined limit, known as alignment with decoupling, in which the Lagrangians $\mathcal{L}_{\phi / a}$ contain all the interactions necessary to predict the $t \bar{t}+E_{T}^{\text {miss }}$ and $t W+E_{T}^{\text {miss }}$ signatures up to terms that formally vanish for infinitely heavy $2 \mathrm{HDM}$ spin-0 states $H, A, H^{ \pm}$. There are thus well-motivated UV complete scenarios for which the above interactions provide the dominant contributions to $t \bar{t}+E_{T}^{\text {miss }}$ and $t W+E_{T}^{\text {miss }}$ production (see figure 1 for example Feynman diagrams). As a result (2.1) can also serve as a useful starting point for further phenomenological investigations of the interplay and complementarity of the $t \bar{t}+E_{T}^{\text {miss }}$ and $t W+E_{T}^{\text {miss }}$ channels in certain limits of next-generation spin-0 DM models.

\section{Unitarity considerations}

An issue related to the discussion presented at the end of the last section is whether the production of DM in association with top quarks can be reliable calculated in the context of (2.1). To address this question, we will study the UV behaviour of scattering amplitudes (cf. [23-27] for related discussions). Since the $t \bar{t}+E_{T}^{\text {miss }}$ amplitudes arising from $\mathcal{L}_{\phi / a}$ turn out to be well-behaved in the high-energy limit, the ensuing discussion will entirely focus on the $t W+E_{T}^{\text {miss }}$ signature.

The Feynman graphs that lead to $t W+E_{T}^{\text {miss }}$ production in the spin- $0 s$-channel DM simplified models (2.1) are shown in the lower row of figure 1. Diagrams where the mediator $\phi / a$ is radiated off a bottom quark are suppressed by the bottom Yukawa coupling and will be neglected in the further discussion. In the high-energy limit, the emission of the $W$ boson can be treated in the effective $W$-boson approximation [28, 29]. In this approximation, the process $g b \rightarrow t W^{-} \chi \bar{\chi}$ factorises into the interactions $g b \rightarrow b$ (or $g t \rightarrow t$ ), 
the hard scattering $b \rightarrow t W^{-} \phi / a$ and the decay $\phi / a \rightarrow \chi \bar{\chi}$. This factorisation allows one to study the high-energy behaviour of the process $b \rightarrow t W^{-} \phi / a$ to gain an understanding of the energy growth of the full $g b \rightarrow t W^{-} \chi \bar{\chi}$ amplitude. Our discussion will follow closely the arguments given in [30, 31].

In the high-energy limit, where the Mandelstam variables fulfil $\hat{s},-\hat{t},-\hat{u} \gg m_{t}^{2}, m_{W}^{2}, m_{\phi}^{2}$ and for a longitudinal-polarised $W$ boson, the $b \rightarrow t W^{-} \phi$ amplitude takes the following form in the scalar $s$-channel DM simplified model:

$$
\mathcal{A}_{\phi}=V_{t b}^{*} \frac{y_{t}}{v} g_{t} \bar{b} P_{L} t
$$

Here $V_{t b}$ is the relevant Cabibbo-Kobayashi-Maskawa matrix element and $P_{L}=\left(1-\gamma_{5}\right) / 2$ projects onto left-handed fields. In the case of the pseudoscalar mediator $a$, one instead finds $\mathcal{A}_{a}=-i \mathcal{A}_{\phi}$.

The above results imply that the $s$-wave amplitude $a_{0}$ of the $b \rightarrow t W^{-} \phi / a$ process grows linearly with the partonic centre-of-mass $(\mathrm{CM})$ energy $\hat{E}_{\mathrm{CM}}=\sqrt{\hat{s}}$. Explicitly, we find that

$$
\left|a_{0}\right|=\frac{\left|V_{t b}^{*}\right|}{24 \pi} \frac{y_{t}}{v} g_{t} \hat{E}_{\mathrm{CM}},
$$

which allows to estimate the cut-off scale $\Lambda$ where perturbative unitarity is lost. Imposing the condition $\left|a_{0}\right|<1$ and identifying $\hat{E}_{\mathrm{CM}} \simeq \Lambda$, the relation (3.2) leads to

$$
\Lambda \simeq \frac{24 \pi}{\left|V_{t b}^{*}\right|} \frac{v}{y_{t}} \frac{1}{g_{t}} \simeq \frac{18.6 \mathrm{TeV}}{g_{t}}
$$

where to obtain the final result we have used that $V_{t b} \simeq 1$ and $y_{t} \simeq 1$. In order to make the $g b \rightarrow t W^{-} \chi \bar{\chi}$ amplitudes well-behaved additional particles and/or couplings thus have to appear at a scale around $\Lambda$, allowing to embed (2.1) into a gauge-invariant UV complete theory.

While the question to which extent these new states and/or interactions will modify the DM phenomenology is a model-dependent issue, the relation (3.3) can be used to determine whether the $t W+E_{T}^{\text {miss }}$ signature can be reliably computed in the framework of spin-0 $s$-channel DM simplified models (2.1). To do this, we study the dependence of the fiducial $t W+E_{T}^{\text {miss }}$ cross sections on the partonic CM energy, employing the experimental selection that is used in section 6 to disentangle the $t W+E_{T}^{\text {miss }}$ signal from the SM background. The corresponding signal region is called $\mathrm{SR}_{t W}$ and the relevant cuts can be found in table 1 .

In figure 2 we present normalised distributions for scalar (left) and pseudoscalar (right) mediators of type (2.1) obtained with the MC setup described in section 5. The shown predictions correspond to $p p$ collisions at $14 \mathrm{TeV}$ and the ATLAS/CMS DM Forum (DMF) [2, $3]$ benchmark parameters $m_{\chi}=1 \mathrm{GeV}$ and $g_{\chi}=g_{t}=1$. One observes that for a mediator mass of $100 \mathrm{GeV}(500 \mathrm{GeV})$ the spectra of the normalised fiducial cross sections have peaks at around $700 \mathrm{GeV}(1.1 \mathrm{TeV})$ and tails that rapidly fall off for increasing $\hat{E}_{\mathrm{CM}}$. Numerically, we find for example that the phase-space region with $\hat{E}_{\mathrm{CM}}>3 \mathrm{TeV}$ contributes only a fraction of $0.2 \%$ to $1.2 \%$ to the fiducial $t W+E_{T}^{\text {miss }}$ cross sections for the model realisations considered in the figure. The fraction of $t W+E_{T}^{\text {miss }}$ events with partonic CM energies at or above the cut-off (3.3) is hence negligible at $14 \mathrm{TeV}$. In consequence, the perturbative 

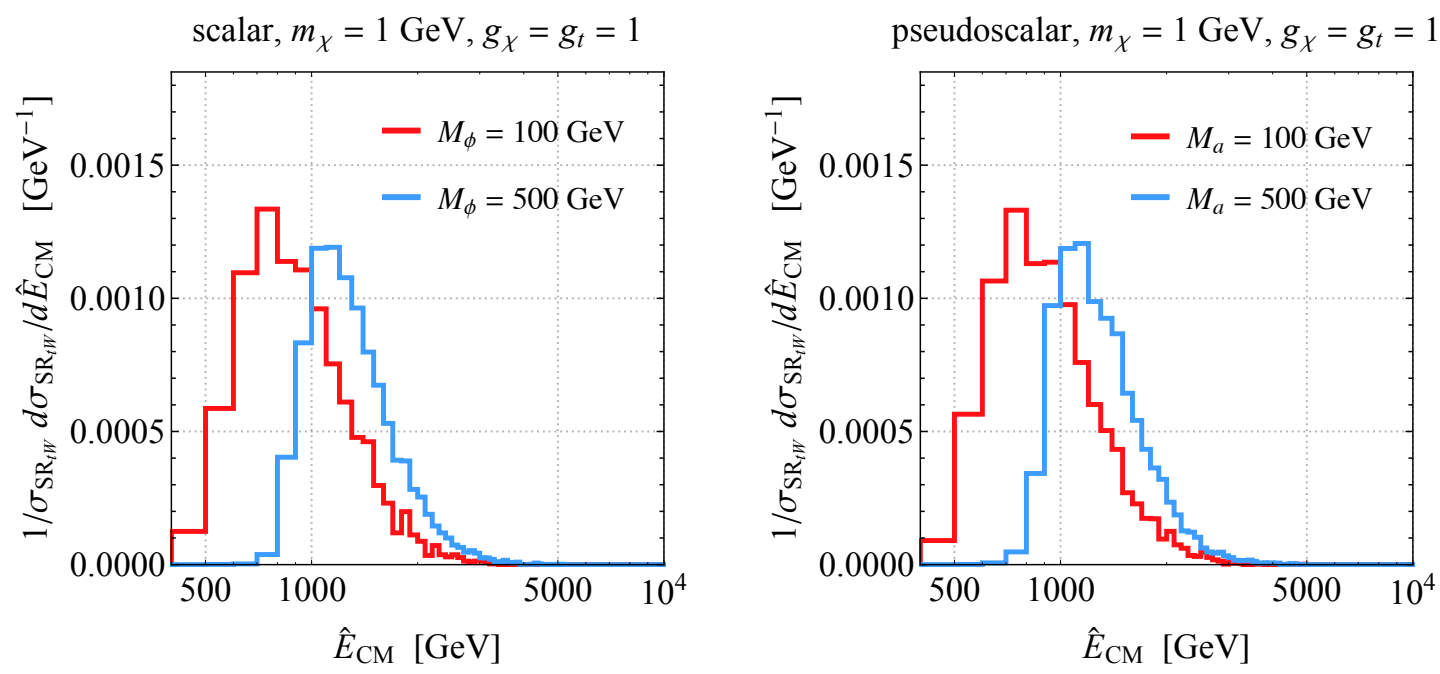

Figure 2. Normalised differential fiducial cross sections for $t W+E_{T}^{\text {miss }}$ production as a function of the partonic CM energy in the scalar (left) and pseudoscalar (right) $s$-channel DM simplified model. The shown predictions correspond to $14 \mathrm{TeV} p p$ collisions, the selections $\mathrm{SR}_{t W}$ defined in table 1 and the parameter choices indicated in the legends and the headlines of the two panels.

computation of the $t W+E_{T}^{\text {miss }}$ signal can be fully trusted, and unitarity violation in the production of DM in association with top quarks is spurious at LHC energies in the spin-0 $s$-channel DM simplified models (2.1).

\section{Angular correlations of the $t W+E_{T}^{\text {miss }}$ signal}

In the case of the $t \bar{t}+E_{T}^{\text {miss }}$ signal, it has been shown in $[8,10]$ that information on the spin0 mediator type is encoded in the correlations between the final-state top quarks as well as their decay products. As explained in the publication [10], the observed angular correlations in $t \bar{t}+E_{T}^{\text {miss }}$ production can be understood from the energy behaviour of the fragmentation function $f_{t \rightarrow \phi / a}$ that describes the radiation of $\phi / a$ fields from top quarks. In the case of the interactions (2.1), the leading (universal) fragmentation functions read $[32,33]$

$$
f_{t \rightarrow \phi}=\frac{g_{t}^{2}}{(4 \pi)^{2}}\left[\frac{4(1-x)}{x}+x \ln \left(\frac{\hat{s}}{m_{t}^{2}}\right)\right], \quad f_{t \rightarrow a}=\frac{g_{t}^{2}}{(4 \pi)^{2}}\left[x \ln \left(\frac{\hat{s}}{m_{t}^{2}}\right)\right]
$$

where it is assumed that $\hat{s} \gg 4 m_{t}^{2} \gg M_{\phi / a}^{2}$ and $\sqrt{\hat{s}}=2 \hat{E} / x$ with $\hat{E}$ denoting the energy of the emitted mediator. The first expression in (4.1) implies that a light scalar is radiated off top quarks preferentially with small energy (or equivalent small momentum fraction $x$ ) due to the soft singularity proportional to $1 / x$ in $f_{t \rightarrow \phi}$. This soft-enhanced term is instead absent in the pseudoscalar fragmentation function $f_{t \rightarrow a}$.

The arguments presented in [10] can also be used to gain a qualitative understanding of the angular correlations of the $t W+E_{T}^{\text {miss }}$ signal (see also [34] for a related discussion). Below we will focus on $\cos \theta_{t W}=\tanh \left(\Delta \eta_{t W} / 2\right)$, because in the work [10] the related observable $\cos \theta_{t \bar{t}}$ has been shown to be a good CP analyser in the case of the $t \bar{t}+E_{T}^{\text {miss }}$ signature. Here $\Delta \eta_{t W}$ denotes the difference in pseudorapidity of the top quark and the 

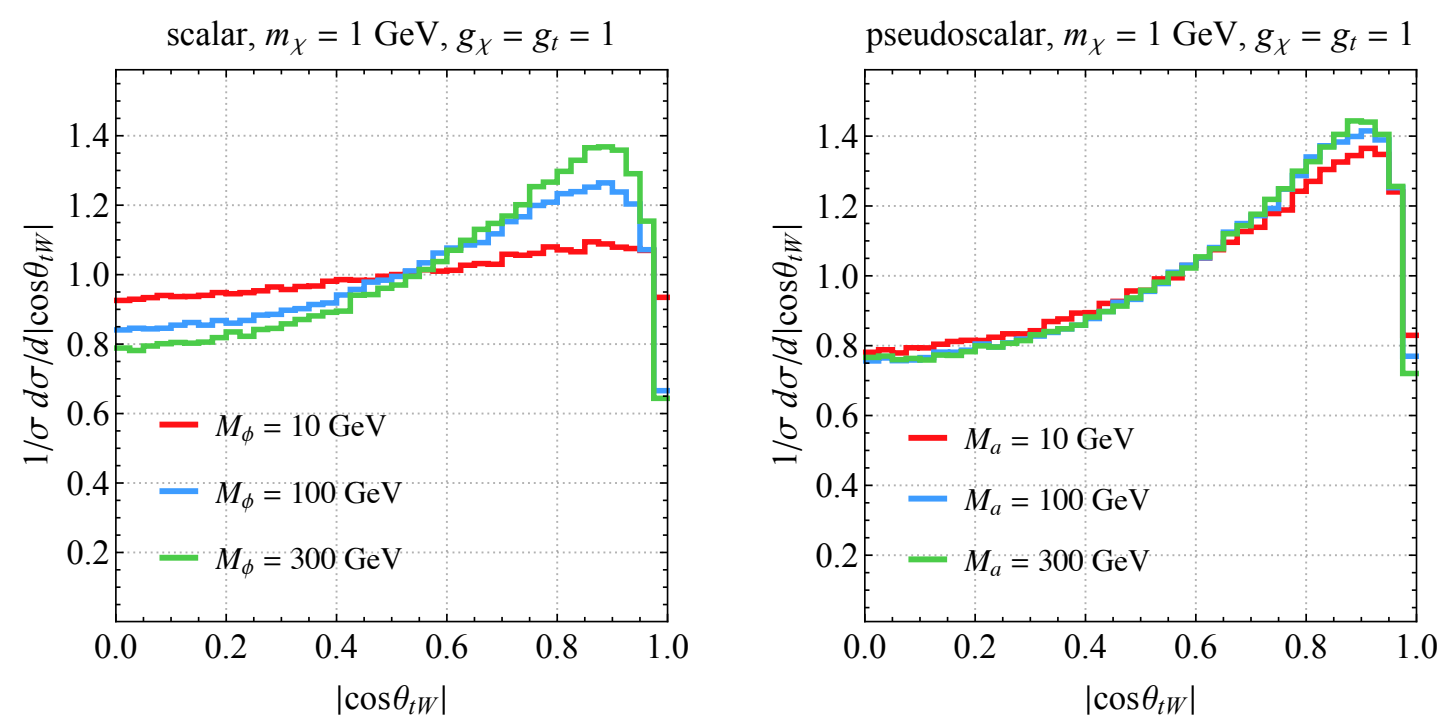

Figure 3. Normalised $\left|\cos \theta_{t W}\right|$ distributions for the $t W+E_{T}^{\text {miss }}$ signature in the scalar (left) and pseudoscalar (right) $s$-channel DM simplified model. The shown results correspond to the $14 \mathrm{TeV}$ LHC and the parameters given in the legends and headlines of the figure.

$W$ boson. All spectra shown in this subsection have been obtained with the MC setup described in section 5, correspond to $p p$ collisions at $14 \mathrm{TeV}$ and employ the DMF benchmark parameter choices $m_{\chi}=1 \mathrm{GeV}$ and $g_{\chi}=g_{t}=1$. We emphasise that the shapes of the angular correlations discussed below as well as in section 6 are independent of the specific choices made for $m_{\chi}, g_{\chi}$ and $g_{t}$. This feature can be understood by noting that the two considered DM signatures factorise into $p p \rightarrow t \bar{t}+\phi / a$ or $p p \rightarrow t W+\phi / a$ production followed by $\phi / a \rightarrow \chi \bar{\chi}$. As a result of this factorisation the normalised kinematic distributions of the $t \bar{t}$ or $t W$ systems as well as their decay products do only depend on the mass $M_{\phi / a}$ relevant for on-shell production and decay but not on the other spin-0 s-channel DM simplified model parameters. We finally mention that we have explicitly verified the parameter independence of the normalised angular correlations of the $t \bar{t}+E_{T}^{\text {miss }}$ and $t W+E_{T}^{\text {miss }}$ signal by generating $\mathrm{MC}$ events using $M_{\phi / a}=300 \mathrm{GeV}, g_{\chi}=g_{t}=1$ and $m_{\chi}=100 \mathrm{GeV}$ instead of $m_{\chi}=1 \mathrm{GeV}$.

In figure 3 we present results for the $\left|\cos \theta_{t W}\right|$ distributions in the $t W+E_{T}^{\text {miss }}$ channel for three different realisations of the scalar and pseudoscalar $s$-channel DM simplified models (2.1). The displayed spectra are all normalised to unity and no selection cuts have been imposed. One sees that for a very light mediator of $10 \mathrm{GeV}$ (red curves) the normalised $\left|\cos \theta_{t W}\right|$ spectrum is almost flat in the scalar case, while it is enhanced toward larger values of $\left|\cos \theta_{t W}\right|$ for a pseudoscalar. The flatness of the scalar spectrum is a result of the $1 / x$ singularity of the fragmentation function $f_{t \rightarrow \phi}$ (cf. (4.1)), which favours emissions of soft $\phi$ fields. Compared to a pseudoscalar, a scalar of the same mass hence tends to be produced more forward, and in consequence the accompanying top quark and $W$ boson are produced more central. On average the observable $\left|\cos \theta_{t W}\right|$ is thus smaller for a very light scalar than a pseudoscalar. For heavier mediators of $100 \mathrm{GeV}$ (blue curves) the soft enhancement of $f_{t \rightarrow \phi}$ is less important, and the $\left|\cos \theta_{t W}\right|$ spectra all de- 

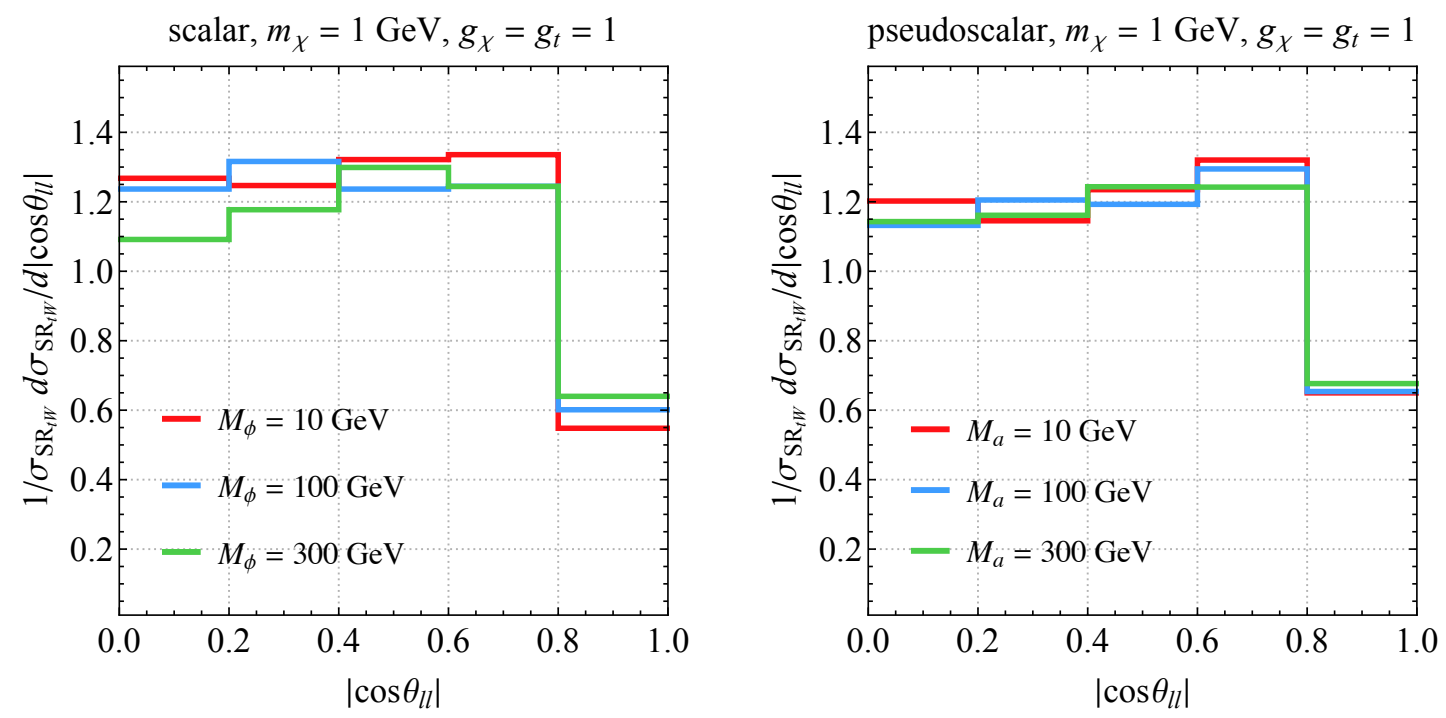

Figure 4. Normalised $\left|\cos \theta_{l l}\right|$ distributions corresponding to $t W+E_{T}^{\mathrm{miss}}$ production with subsequent decays $t \rightarrow b W \rightarrow b l \nu$ and $W \rightarrow l \nu$. The shown results correspond to $p p$ collisions at $14 \mathrm{TeV}$ and adopt apart from $C_{\mathrm{em}}>150 \mathrm{GeV}$ the experimental selections $\mathrm{SR}_{t W}$ as given in table 1 . The parameter choices of the scalar (left) and pseudoscalar (right) $s$-channel DM simplified models are indicated in the panels.

velop a peak close to 1 irrespectively of the mediator type. The peaks become more pronounced and the shapes more similar when the mediator mass is increased further to $300 \mathrm{GeV}$ (green curves).

The results presented above suggest that for light spin- 0 mediators angular correlations in the $t W$ system can be used to study the CP properties of the mediation mechanism in $t W+E_{T}^{\text {miss }}$ production. The directions of the top quark and the $W$ boson are, however, experimentally not directly accessible, since the dilepton final state contains four invisible particles (i.e. two neutrinos and two DM particles). The relative orientation of the top quark and the $W$ boson has hence to be obtained indirectly from measurements of the angular distributions of the charged-lepton pairs resulting from $t \rightarrow b W \rightarrow b l \nu$ and $W \rightarrow l \nu$. The crucial question is whether the discriminating power of angular variables such as $\cos \theta_{l l}$ survives the experimental cuts necessary to extract the DM signal from the SM backgrounds. To address this issue we show in figure 4 normalised $\left|\cos \theta_{l l}\right|$ distributions of the $t W+E_{T}^{\text {miss }}$ signature, imposing apart from $C_{\mathrm{em}}>150 \mathrm{GeV}$ the selection requirements $\mathrm{SR}_{t W}$ as specified in table 1. We again display results for the three mediator masses $10 \mathrm{GeV}, 100 \mathrm{GeV}$ and $300 \mathrm{GeV}$. One observes that the signal selections tend to wash out shape differences in the distributions of the $\left|\cos \theta_{l l}\right|$ variable, thereby reducing the sensitivity of this observable in realistic experimental analyses of the $t W+E_{T}^{\text {miss }}$ signal. Compared to the $t \bar{t}+E_{T}^{\text {miss }}$ case analysed in detail in the work [10], angular correlations of the $t W+E_{T}^{\text {miss }}$ signal alone thus turn out to be less useful as a model discriminant. However, we will see later that the observable $\left|\cos \theta_{l l}\right|$ still provides sensitivity to the mass and the $\mathrm{CP}$ nature of the mediating particle if the dilepton events resulting from the $t \bar{t}+E_{T}^{\text {miss }}$ and $t W+E_{T}^{\text {miss }}$ channels are combined into a single signal sample as done in section 6 . 


\section{MC generation and detector simulation}

All the signal samples shown in this article are generated at next-to-leading order (NLO) using a slightly modified version of the DMsimp implementation [7] of the Lagrangians (2.1) together with MadGraph5_aMC@NLO [35] and NNPDF3.0 parton distribution functions (PDFs) [36]. The final-state top quarks and $W$ bosons are decayed with MadSpin [37] and the events are showered with PYTHIA 8.2 [38]. We produce both the $t \bar{t}+E_{T}^{\text {miss }}$ and the $t W+E_{T}^{\text {miss }}$ final states assuming $p p$ collisions at $14 \mathrm{TeV}$. Following [39, 40], we avoid double counting in the $t W+E_{T}^{\text {miss }}$ channel by removing all doubly resonant diagrams involving top quarks, employing the so-called diagram removal procedure [41]. We consider nine different values of the mediator masses $M_{\phi / a}$, varying from $10 \mathrm{GeV}$ to $1 \mathrm{TeV}$. The mass of the DM particles is set to $m_{\chi}=1 \mathrm{GeV}$ and we employ $g_{\chi}=g_{t}=1$ for the mediator couplings to DM and to top quarks. Given the strong Yukawa suppression the mediator couplings to the bottom quark and all other light fermions are set to zero. This simplification has no impact on the results obtained in our work. The widths of the mediators are assumed to be minimal and calculated at tree level using MadGraph5_aMC@NLO. Since in the narrow width approximation the signal predictions factorise into the cross sections for $p p \rightarrow t \bar{t} / t W+\phi / a$ production times the $\phi / a \rightarrow \chi \bar{\chi}$ branching ratio, changing the mediator width leads only to a rescaling of the signal strength. The experimental acceptance is instead insensitive to the mediator width, and therefore it is sufficient to generate samples for a single coupling choice. The predictions for other coupling values can then be simply obtained by scaling with the corresponding $\phi / a \rightarrow \chi \bar{\chi}$ branching ratio that dictates the fraction of invisible decays of the mediators.

To model the $t \bar{t}+E_{T}^{\text {miss }}$ and $t W+E_{T}^{\text {miss }}$ backgrounds accurately, SM processes involving at least two leptons coming from the decay of vector bosons are generated. Backgrounds either with fake electrons (e) from jet misidentification or with real non-isolated leptons from the decay of heavy-flavoured hadrons are not considered in our analysis. A reliable estimate of these backgrounds depends on a detailed simulation of the detector effect beyond the scope of this paper. This simplification is motivated by the results of the ATLAS analysis [42], which addresses kinematic configurations similar to the ones targeted in the present study, and finds that the background from non-prompt leptons does not exceed the level of $15 \%$ of the total background. The backgrounds from $t \bar{t}$ [43], $t W[44], W W$, $W Z$ and $Z Z$ production [45, 46] were all generate at NLO with POWHEG BOX [47]. The $Z+$ jets samples are generated at leading order (LO) with MadGraph5_aMC@NLO and contain up to four jets. MadGraph5_aMC@NLO is also used to simulate the $t \bar{t} V$ backgrounds with $V=W, Z$ at LO with a multiplicity of up to two jets. All partonic events are showered with PYTHIA 8.2. The samples produced with POWHEG BOX are normalised to the NLO cross section given by the generator, except $t \bar{t}$ which is normalised to the cross section obtained at next-to-next-to-leading order (NNLO) plus next-to-next-to-leading logarithmic accuracy $[48,49]$. The $V+$ jets samples are normalised to the known NNLO cross sections $[50,51]$, while in the case of the $t \bar{t} V$ samples the NLO cross sections calculated with MadGraph5_aMC@NLO are used as normalisations. 
The actual analysis uses experimentally identified electrons, muons $(\mu)$, photons, jets and $E_{T \text {,miss. }}$. These objects are constructed from the stable particles in the generator output. Jets are obtained by clustering the true momenta of all particles but muons that interact in the calorimeters. Fast Jet [52] is used to construct anti- $k_{t}$ jets [53] of radius $R=0.4$. Jets originating from the hadronisation of bottom quarks ( $b$-jets) are experimentally tagged in the detector (b-tagged). The variable $\vec{p}_{T \text {,miss }}$ with magnitude $E_{T, \text { miss }}$ is defined at truth level, i.e. before applying detector effects, as the vector sum of the transverse momenta of all the invisible particles (neutrinos and DM particles in our case). The effect of the detector on the kinematic variables used in the analysis is simulated by smearing with Gaussian functions the momenta of the reconstructed objects, and by applying reconstruction and tagging efficiency factors tuned to mimic the performance of the ATLAS detector $[54,55]$. More details on our detector simulation can be found in [10, 40].

\section{Analysis strategy}

In order to search for the $t \bar{t}+E_{T}^{\text {miss }}$ and $t W+E_{T}^{\text {miss }}$ signatures, we consider two-lepton final states. This final state can arise from either two top-quark decays $(t \rightarrow b W \rightarrow b l \nu)$ or a top-quark decay and a $W$-boson decay $(W \rightarrow l \nu)$. In both cases, the dominant backgrounds turn out to be $t \bar{t}$ and $t \bar{t} Z$. Since the amount of $E_{T}^{\text {miss }}$ is typically larger for the signal due to the undetected DM and the $m_{T 2}$ observable $[56,57]$ has a sharp edge at $M_{W}$ in the case of $t \bar{t}$ production, these two variables provide the main handles to suppress the leading SM backgrounds. An analysis based on $E_{T}^{\text {miss }}$ and $m_{T 2}$ has been shown in [10, 42] to achieve a good separation of the $t \bar{t}+E_{T}^{\text {miss }}$ signal from the $t \bar{t}$ background, whereas a part of $t \bar{t} Z$ is irreducible. Applying the same strategy to $t W+E_{T}^{\text {miss }}$ production would also allow to separate the signal from the $t \bar{t}$ background, but, due to the lower cross section, it would be difficult to extract the $t W+E_{T}^{\text {miss }}$ signal from the irreducible $t \bar{t} Z$ background. Moreover, it would be complicated to separate $t W+E_{T}^{\text {miss }}$ production from the much larger $t \bar{t}+E_{T}^{\text {miss }}$ signal.

An important ingredient of a realistic analysis strategy thus consists in separating events that are associated to two semileptonic top-quark decays from those with one semileptonic top-quark decay and one leptonic $W$-boson decay. In the former case, the resulting events have two $b$-jets, while in the latter case only a single $b$-jet is produced. Vetoing the second $b$-jet, however, turns out to be an ineffective strategy, as the typical $b$-jet tagging efficiency of $70 \%$ to $80 \%$ would result in a large surviving $t \bar{t}$ background. Furthermore, $t W$ production where a bottom quark is extracted from the proton is often accompanied by an additional $b$-jet, and as a result this type of SM background cannot be tamed by vetoing the presence of a second $b$-tagged jet in the event.

Instead of using a $b$-jet veto, our analysis strategy is therefore based on the observation that if a $b$-jet is produced in the top-quark decay, its invariant mass is bounded from above by $\sqrt{m_{t}^{2}-M_{W}^{2}} \simeq 153 \mathrm{GeV}$. Events compatible with two semileptonic top-quark decays can thus be selected or rejected by introducing the observable

$$
m_{b l}^{t}=\min \left(\max \left(m_{l_{1} j_{a}}, m_{l_{2} j_{b}}\right)\right),
$$




\begin{tabular}{|l|c|c|}
\hline & \multicolumn{1}{|c|}{$\mathrm{SR}_{t \bar{t}}$} & $\mathrm{SR}_{t W}$ \\
\hline$N_{l}$ & $=2, \quad p_{T, l_{1}}>25 \mathrm{GeV}, \quad p_{T, l_{2}}>20 \mathrm{GeV}, \quad\left|\eta_{l}\right|<2.5$ \\
$m_{l l}$ & $>20 \mathrm{GeV}, \quad Z$-boson veto for opposite-sign leptons \\
$N_{b}$ & \multicolumn{2}{|c|}{$>0, \quad p_{T, b}>30 \mathrm{GeV}, \quad\left|\eta_{b}\right|<2.5$} \\
$m_{T 2}$ & \multicolumn{2}{|c|}{$>100 \mathrm{GeV}$} \\
\hline$m_{b l}^{t}$ & $<160 \mathrm{GeV}$ & $>160 \mathrm{GeV} \quad \| \quad N_{j}=1$ \\
\hline$\left|\Delta \phi_{\text {min }}\right|$ & $>0.8$ & $\mathrm{n} / \mathrm{a}$ \\
$\left|\Delta \phi_{\text {boost }}\right|$ & $<1.2$ & $<500 \mathrm{GeV}$ \\
$M_{\text {scal }}$ & $\mathrm{n} / \mathrm{a}$ & $>200 \mathrm{GeV}$ \\
$C_{\text {em }}$ & $>200 \mathrm{GeV}$ & shape fit \\
$\left|\cos \theta_{l l}\right|$ & shape fit & \\
\hline
\end{tabular}

Table 1. Definition of the two signal regions $\mathrm{SR}_{t \bar{t}}$ and $\mathrm{SR}_{t W}$. For further details consult the text.

where $l_{1}$ and $l_{2}$ denote the two final-state leptons and the minimisation runs over all pairs $\left\{j_{a}, j_{b}\right\}$ of jets inside a predefined set of test jets. Based on the number of $b$-jets in the event, the set of test jets is defined as follows. If the event includes one or two $b$-jets, an additional test jet is considered, chosen as the non- $b$-tagged jet with the highest $b$-tagging weight. If three $b$-jets are found, they are all taken as test jets.

Our search strategy is based on two distinct signal regions. A signal region that we call $\mathrm{SR}_{t \bar{t}}$, aimed at separating $t \bar{t}+E_{T}^{\text {miss }}$ production from the dominant $t \bar{t}$ background, and a signal region called $\mathrm{SR}_{t W}$ that is designed to disentangling the $t W+E_{T}^{\text {miss }}$ signal from both $t \bar{t}+E_{T}^{\text {miss }}$ production and the SM background. The definitions of the signal regions are summarised in table 1 . The first part of the selections is common for the two signal regions. In a first step, events with exactly two isolated oppositely charged leptons ( $N_{l}=2$ with $l=e, \mu$ or one of each flavour) are selected. The individual leptons are required to satisfy $p_{T, l_{1}}>25 \mathrm{GeV}, p_{T, l_{2}}>20 \mathrm{GeV},\left|\eta_{l}\right|<2.5$ and their invariant mass has to fulfill $m_{l l}>20 \mathrm{GeV}$. The kinematic requirements on the charged leptons are such that the events of interest will be selected with full efficiency by the dilepton triggers foreseen for LHC Run-3 and the high-luminosity phase of the LHC (HL-LHC), as documented in table 6.4 of [58]. If the charged leptons are of the same flavour the additional requirement $m_{l l} \notin[71,111] \mathrm{GeV}$ is imposed to veto events where the charged lepton pair arises from $Z \rightarrow l^{+} l^{-}$. Furthermore, each event is required to contain at least one $b$-tagged jet $\left(N_{b}>0\right)$ with $p_{T, b}>30 \mathrm{GeV}$ and $\left|\eta_{b}\right|<2.5$. An initial rejection of the SM backgrounds, where $E_{T}^{\text {miss }}$ is due to the neutrinos from the decay of two $W$ bosons, is finally achieved by requiring that $m_{T 2}>100 \mathrm{GeV}$.

The first selection that differs between the two signal regions is a cut on the $m_{b l}^{t}$ observable introduced in (6.1). In order to illustrate the impact of this selection, we show in figure 5 the distributions of $m_{b l}^{t}$ for the $t \bar{t}+E_{T}^{\text {miss }}$ and the $t W+E_{T}^{\text {miss }}$ signals corresponding to a pseudoscalar of mass $M_{a}=100 \mathrm{GeV}, m_{\chi}=1 \mathrm{GeV}$ and $g_{\chi}=g_{t}=1$. The total SM background, which is dominated by $t \bar{t}$ production, is also displayed. For an easy comparison with the DM signals it has been scaled down by a factor of 100 . 


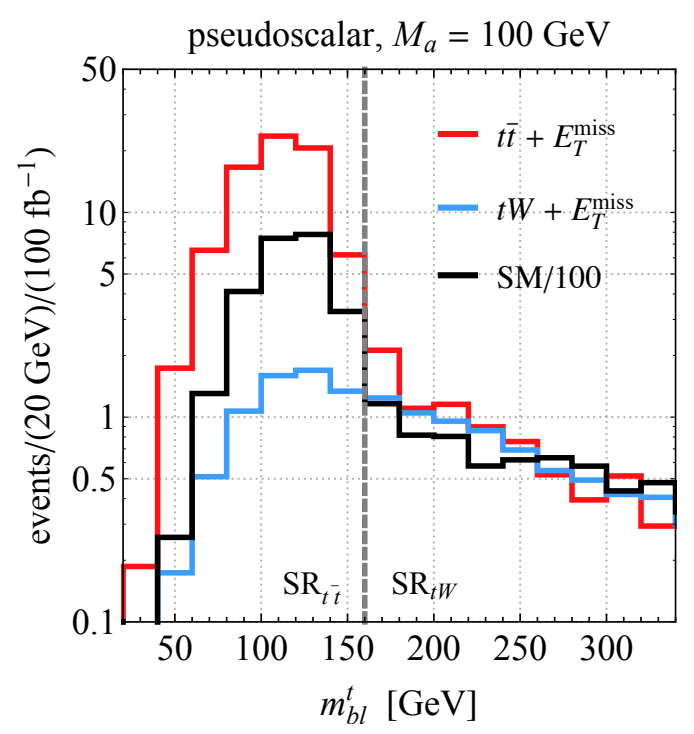

Figure 5. Distributions of the $m_{b l}^{t}$ variable (6.1) for the $t \bar{t}+E_{T}^{\text {miss }}$ signal, the $t W+E_{T}^{\text {miss }}$ signal, and the sum of SM backgrounds scaled down by a factor 100. The shown results correspond to $100 \mathrm{fb}^{-1}$ of $14 \mathrm{TeV}$ LHC data and the common lepton, $b$-jet and $m_{T 2}$ selections summarised in table 1 . The used model parameters are $m_{\chi}=1 \mathrm{GeV}, g_{\chi}=g_{t}=1$ and those appearing in the headline of the figure. The dashed line corresponds to $m_{b l}^{t}=160 \mathrm{GeV}$, which represents the boundary between the two signal regions $\mathrm{SR}_{t \bar{t}}$ and $\mathrm{SR}_{t W}$.

All event samples correspond to $100 \mathrm{fb}^{-1}$ of $14 \mathrm{TeV}$ LHC data and the initial selection requirements as described above have been imposed. From the figure it is clear that the region $m_{b l}^{t}<160 \mathrm{GeV}$ is dominated by the $t \bar{t}+E_{T}^{\text {miss }}$ signal, and is therefore used to define $\mathrm{SR}_{t \bar{t}}$. Numerically, we find for the case at hand that $91 \%$ of the DM signal in $\mathrm{SR}_{t \bar{t}}$ arises from $t \bar{t}+E_{T}^{\text {miss }}$, while only $9 \%$ are due to $t W+E_{T}^{\text {miss }}$. In the region $m_{b l}^{t}>160 \mathrm{GeV}$, one instead obtains similar event fractions of around $50 \%$ for both $t \bar{t}+E_{T}^{\text {miss }}$ and $t W+E_{T}^{\text {miss }}$, and hence our signal region $\mathrm{SR}_{t W}$ adopts this requirement. The variable $m_{b l}^{t}$ is only defined for events with at least two reconstructed jets with $p_{T, j}>25 \mathrm{GeV}$, and events with only one reconstructed jet $\left(N_{j}=1\right)$ are assigned to $\mathrm{SR}_{t W}$. Relative to the $\mathrm{SM}$ background the combined DM signal in $\mathrm{SR}_{t \bar{t}}\left(\mathrm{SR}_{t W}\right)$ amounts to $3.4 \%$ (2.0\%). Notice that since the shape of the $m_{b l}^{t}$ distributions is essentially independent of the type of the mediator, its mass and the other new-physics parameters appearing in (2.1), the $m_{b l}^{t}$ selection can be applied unchanged for each model realisation.

Further selections are used in the two signal regions to optimise the rejection of the SM backgrounds. All reconstructed jets with $p_{\mathrm{T}}^{j}>25 \mathrm{GeV}$ within $\left|\eta_{\ell}\right|<2.5$ have to satisfy $\left|\Delta \phi_{\min }\right|>0.8$ for both signal regions, where $\Delta \phi_{\min }$ corresponds to the angle between $\vec{p}_{\mathrm{T}}^{j}$ and $\vec{p}_{T}^{\text {miss }}$ for the jet closest to $E_{T}^{\text {miss }}$ in the azimuthal plane. The variable $\Delta \phi_{\text {boost }}$ defined as the azimuthal angular distance between $\vec{p}_{T}^{\text {miss }}$ and the vector sum of $\vec{p}_{T}^{\text {miss }}, \vec{p}_{T, l_{1}}$ and $\vec{p}_{T, l_{2}}$, must satisfy the requirement $\left|\Delta \phi_{\text {boost }}\right|<1.2$ for $\mathrm{SR}_{t \bar{t}}$. In the case of the signal region $\mathrm{SR}_{t W}$, we in addition require that the scalar sum $M_{\text {scal }}$ of the transverse momenta of all the jets observed in the event satisfies $M_{\text {scal }}<500 \mathrm{GeV}$. We finally demand that the variable 

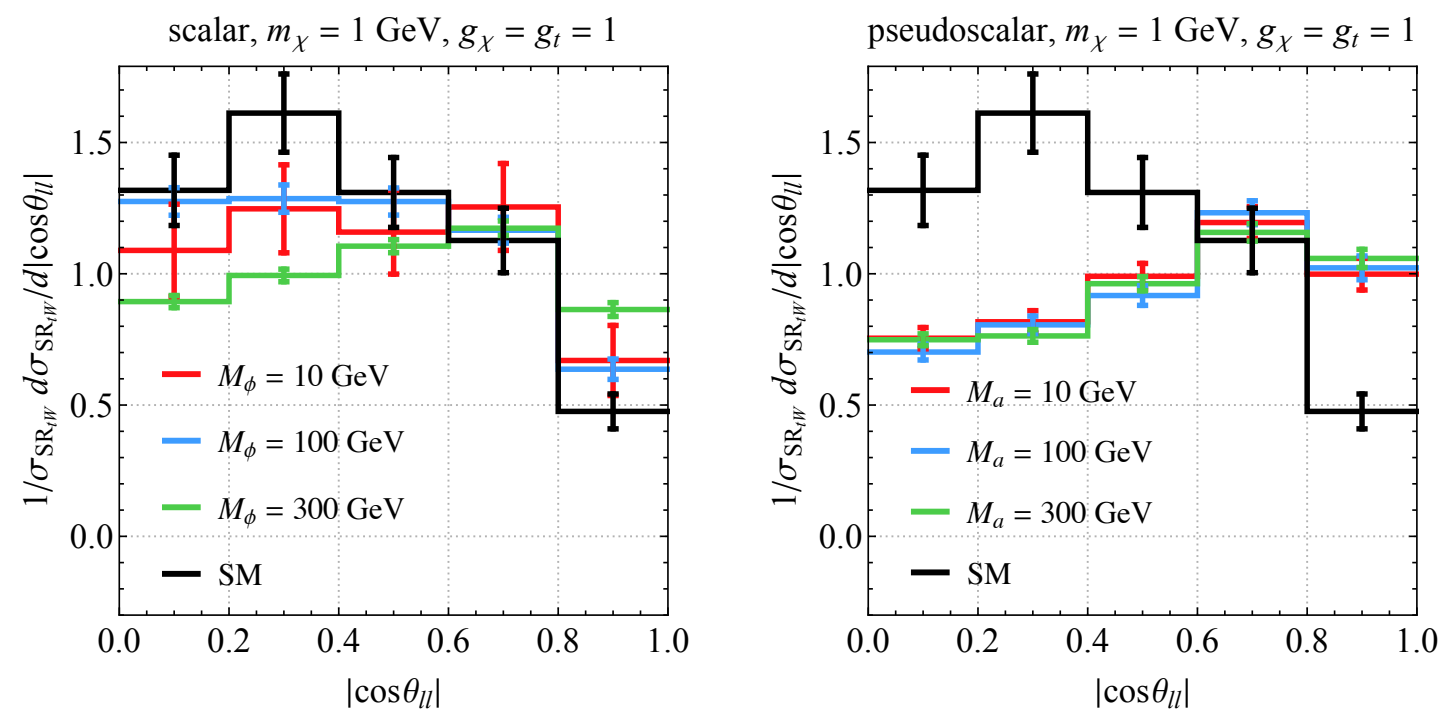

Figure 6. As figure 4 but combining the dilepton events that arise from both $t \bar{t}+E_{T}^{\text {miss }}$ and $t W+E_{T}^{\text {miss }}$ production in spin-0 s-channel DM simplified models into a single sample. The shown error bars represent the statistical errors associated to our MC simulations. For comparison also the SM distribution is depicted.

$C_{\mathrm{em}}=m_{T 2}+0.2 E_{T}^{\mathrm{miss}}$ introduced in [10] exceeds $200 \mathrm{GeV}$ in both signal regions. We will comment further on the impact of this cut at the end of this section.

After impose all of these cuts, the surviving SM background amounts to approximately 30 (20) events for $\mathrm{SR}_{t \bar{t}}\left(\mathrm{SR}_{t W}\right)$ per $300 \mathrm{fb}^{-1}$ of integrated luminosity. The $t \bar{t}+E_{T}^{\text {miss }}$ acceptance in $\mathrm{SR}_{t \bar{t}}$ varies between $0.1 \%(0.5 \%)$ and $1.9 \%$ (1.8\%) for scalar (pseudoscalar) mediators with masses between $100 \mathrm{GeV}$ and $1 \mathrm{TeV}$, while the $t W+E_{T}^{\text {miss }}$ acceptance in $\mathrm{SR}_{t W}$ amounts to between $0.7 \%(1.1 \%)$ and $3.6 \%$ (3.5\%) for scalar (pseudoscalar) mediators in the same mass range. The fraction of $t W+E_{T}^{\text {miss }}$ events in $\mathrm{SR}_{t \bar{t}}\left(\mathrm{SR}_{t W}\right)$ turns out to be between $8 \%(50 \%)$ and $20 \%(75 \%)$ for $M_{\phi / a}$ values in the range of $10 \mathrm{GeV}$ to $1 \mathrm{TeV}$, and almost independent of the $\mathrm{CP}$ nature of the mediator. These numbers imply that our analysis strategy indeed achieves the goal of separating the DM signal into two viable signal regions with very different relative contributions from $t \bar{t}+E_{T}^{\text {miss }}$ and $t W+E_{T}^{\text {miss }}$ production. In the range of $10 \mathrm{GeV}$ to $1 \mathrm{TeV}$, the ratio of the number of signal events in $\mathrm{SR}_{t \bar{t}}$ and $\mathrm{SR}_{t W}$ decreases monotonically with mediator mass from around 2.0 (1.5) to 0.8 (1.0) in the scalar (pseudoscalar) case. After a LHC discovery, measurements of this ratios together with studies of the $\left|\cos \theta_{l l}\right|$ distribution [10] will therefore provide useful handles to determining the properties of the spin- 0 mediators such as mass and $\mathrm{CP}$ quantum number.

Having developed a realistic strategy for the detection of production of DM plus top quarks, we can now come back to the issue of angular correlations of the DM signal. Since in the signal region $\mathrm{SR}_{t W}$ the processes $t \bar{t}+E_{T}^{\text {miss }}$ and $t W+E_{T}^{\text {miss }}$ contribute with similar weight, we show in figure 6 normalised $\left|\cos \theta_{l l}\right|$ distributions of the combined $t \bar{t}+$ $E_{T}^{\mathrm{miss}}$ and $t W+E_{T}^{\mathrm{miss}}$ signature corresponding, apart from $C_{\mathrm{em}}>150 \mathrm{GeV}$, to the $\mathrm{SR}_{t W}$ selection (cf. table 1). Compared to the results presented in figure 4, one observes that the shape differences in the spectra of the $\left|\cos \theta_{l l}\right|$ variable are more pronounced once the 


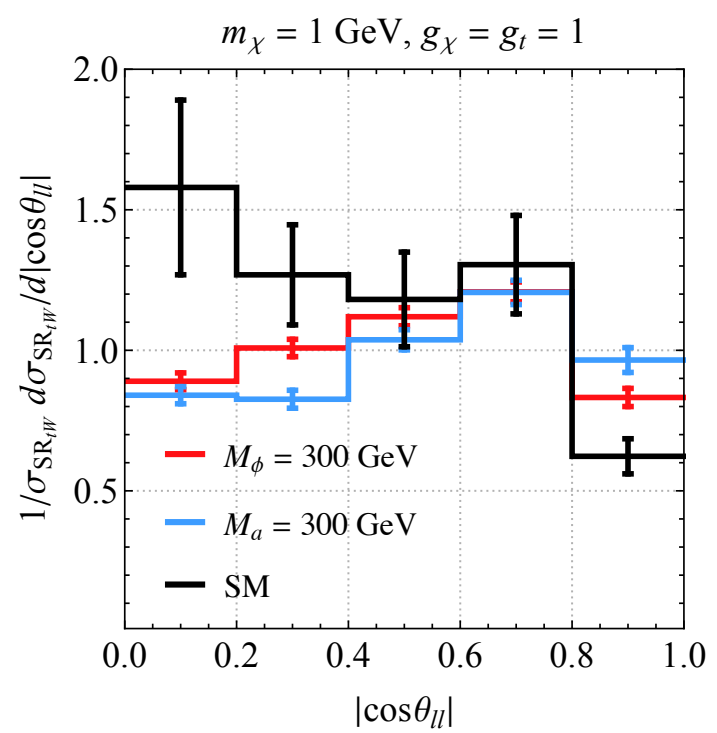

Figure 7. Normalised $\left|\cos \theta_{l l}\right|$ distributions of the combined $t \bar{t}+E_{T}^{\text {miss }}$ and $t W+E_{T}^{\text {miss }}$ signal and the SM background at the $14 \mathrm{TeV}$ LHC. The depicted results impose the selections $\mathrm{SR}_{t W}$ summarised in table 1 . The used parameter choices for the $s$-channel DM simplified models are indicated in the legend and the headline of the panel and the error bars represent the statistical errors associated to our MC simulations.

dilepton events resulting from $t \bar{t}+E_{T}^{\text {miss }}$ have been added to those coming from $t W+$ $E_{T}^{\text {miss }}$. The observable $\left|\cos \theta_{l l}\right|$ therefore still shows promising discriminating properties in a realistic experimental analysis (such as the one proposed here based on the signal region $\mathrm{SR}_{t W}$ ) where two-lepton events will always arise from the sum of $t \bar{t}+E_{T}^{\mathrm{miss}}$ and $t W+E_{T}^{\text {miss }}$ production.

To further stress the latter point, we compare in figure 7 the normalised $\left|\cos \theta_{l l}\right|$ spectra of the SM background with the combined DM signal for a scalar and pseudoscalar mediator of $300 \mathrm{GeV}$. The depicted results correspond to differential cross sections in the fiducial region $\mathrm{SR}_{t W}$. The figure demonstrates that a shape fit to $\left|\cos \theta_{l l}\right|$ in the signal region $\mathrm{SR}_{t W}$ will enhance the search sensitivity for the combination of $t \bar{t}+E_{T}^{\mathrm{miss}}$ and $t W+$ $E_{T}^{\text {miss }}$ production. In our actual analysis, we therefore perform a shape fit to the $\left|\cos \theta_{l l}\right|$ observable in both signal regions. While such a shape fit has already been employed in [10] for a $\mathrm{SR}_{t \bar{t}}$-like event selection, in the case of $\mathrm{SR}_{t W}$ it is a new ingredient of our search strategy.

Before presenting the future constraints on the parameter space of the spin- $0 s$-channel DM simplified models, we add that a shape fit to $C_{\mathrm{em}}$ would increase the experimental reach in both signal regions. The obtained improvements depend, however, strongly on the assumed uncertainty on the shape of the sharply falling SM spectrum of $C_{\mathrm{em}}$. This uncertainty itself depends on the experimental techniques used to estimate the backgrounds, and can hence only be determined reliable within the context of an analysis based on real data. We therefore prefer to use a fixed cut on $C_{\mathrm{em}}$ in both search regions since it is the simplest choice, but still allows to illustrate the interplay of the two signal regions $\mathrm{SR}_{t \bar{t}}$ and $\mathrm{SR}_{t W}$ in searching for production of $\mathrm{DM}$ in association with top quarks. We finally 

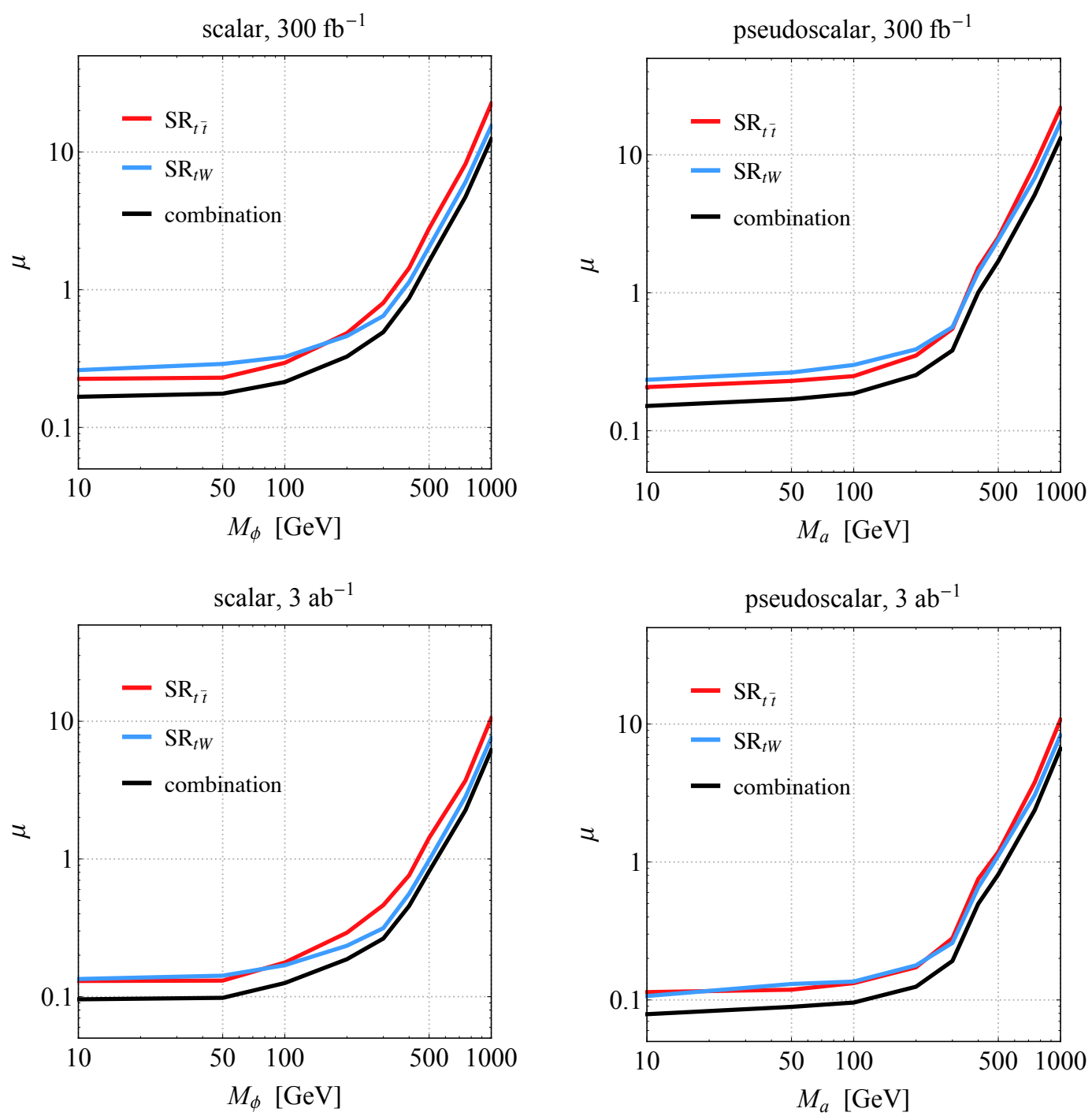

Figure 8. Value of the signal strength $\mu$ that can be excluded at 95\% CL as a function of the mass for scalar (left) and pseudoscalar (right) mediators. The reach for $300 \mathrm{fb}^{-1}$ (upper row) and $3 \mathrm{ab}^{-1}$ (lower row) of $14 \mathrm{TeV}$ LHC data is shown and the parameter choices $m_{\chi}=1 \mathrm{GeV}$ and $g_{\chi}=g_{t}=1$ have been employed.

emphasise that the cut $C_{\mathrm{em}}>200 \mathrm{GeV}$ imposed in both signal regions (see table 1) is not the optimal choice for all model realisations. For light (heavy) spin-0 $s$-channel mediators a weaker (stronger) $C_{\mathrm{em}}$ selection would lead to improved limits.

\section{$7 \quad$ Results}

A profiled likelihood ratio test statistic is used to evaluate the upper limit on the ratio of the signal yield to that predicted in the spin-0 s-channel DM simplified models (2.1). This ratio will be called signal strength in the following and denoted by $\mu$. The $C L_{s}$ method [59] as implemented in the RooStats toolkit [60] is used to derive exclusion limits at $95 \%$ confidence level (CL). Figure 8 displays our limits for integrated luminosities of $300 \mathrm{fb}^{-1}$ (upper 
row) and $3 \mathrm{ab}^{-1}$ (lower row) as a function of the mediator mass for scalar (left) and pseudoscalar (right) mediators. The standard parameter choices $m_{\chi}=1 \mathrm{GeV}$ and $g_{\chi}=g_{t}=1$ are employed. The shown results assume a normalisation uncertainty of $5 \%(20 \%)$ for the DM signal (SM background). As already mentioned in section 6, in both signal regions a shape fit to the $\left|\cos \theta_{l l}\right|$ distribution is performed using five bins with a width of 0.2 . A binby-bin uncertainty of $5 \%$ on the shape of the distribution is assumed. One observes that the search strategy $\mathrm{SR}_{t \bar{t}}$ tends to perform better than $\mathrm{SR}_{t W}$ for mediator masses below approximately $150 \mathrm{GeV}$, while for higher values of $M_{\phi, a}$ the signal region $\mathrm{SR}_{t W}$ typically provides the more stringent bounds. Since the two signal regions are designed without overlap, they can be statistically combined, and the combination yields a notable improvement in reach for both mediator types and over the entire mass range considered. In fact, our combined analysis leads in the scalar case to the $95 \%$ CL limit $M_{\phi}>417 \mathrm{GeV}\left(M_{\phi}>532 \mathrm{GeV}\right)$ at $300 \mathrm{fb}^{-1}\left(3 \mathrm{ab}^{-1}\right)$, which represents a relative improvement of $26 \%$ (22\%) compared to the $\mathrm{SR}_{t \bar{t}}$ bound. For pseudoscalar mediators, the corresponding numbers read $M_{a}>399 \mathrm{GeV}$ $\left(M_{a}>529 \mathrm{GeV}\right)$ at $300 \mathrm{fb}^{-1}\left(3 \mathrm{ab}^{-1}\right)$, implying a relative improvement of $15 \%(16 \%)$ with respect to the $\mathrm{SR}_{t \bar{t}}$ analysis alone. Compared to the earlier studies $[10,11,13,14]$ the proposed combination of the $t \bar{t}+E_{T}^{\text {miss }}$ and $t W+E_{T}^{\text {miss }}$ signals hence provides a significantly improved coverage of the parameter space of spin-0 $s$-channel DM simplified models at both LHC Run-3 and the HL-LHC.

\section{Conclusions}

The goal of this article was to reassess the future sensitivity of the LHC to the production of DM in association with top quarks in the framework of $s$-channel DM simplified models with scalar and pseudoscalar mediators. We have focused on final states with large amounts of $E_{T}^{\text {miss }}$ and two charged leptons. Such configurations can arise either from $t \bar{t}+E_{T}^{\text {miss }}$ production followed by two $t \rightarrow b W \rightarrow b l \nu$ decays or from the $t W+E_{T}^{\text {miss }}$ channel through a $t \rightarrow b W \rightarrow b l \nu$ decay and a $W \rightarrow l \nu$ decay. Given the two different production mechanisms leading to the same final state, the two DM signatures generically overlap and should be treated together in an experimental analysis $[11,12]$.

In order to allow for a theoretically meaningful combination of the $t \bar{t}+E_{T}^{\text {miss }}$ and $t W+E_{T}^{\text {miss }}$ channels in the context of spin-0 $s$-channel DM simplified models, we have first shown that both signatures can be reliable calculated at LHC energies. To this purpose, we have analysed the UV behaviour of the relevant scattering amplitudes. While the $t \bar{t}+E_{T}^{\text {miss }}$ amplitude is well-behaved, the $s$-wave contribution to $t W+E_{T}^{\text {miss }}$ production is found to diverge linearly with the partonic CM energy. The associated UV cut-off where perturbative unitarity breaks down turns out to be in the multi-TeV range for weaklycoupled models. The fraction of $t W+E_{T}^{\text {miss }}$ events with such large partonic CM energies is however negligible for $p p$ collisions at $14 \mathrm{TeV}$ (cf. figure 2). Unitarity violation in the production of DM in association with top quarks is therefore spurious at the LHC in spin-0 $s$-channel DM simplified models.

We have then studied the angular correlations of the $t W+E_{T}^{\text {miss }}$ signature focusing on the observable $\cos \theta_{t W}\left(\cos \theta_{l l}\right)$ that measures the difference in pseudorapidity of the 
top-quark and the $W$ boson (the two charged leptons). In the case of $\cos \theta_{t W}$, we have found that for not too heavy spin-0 particles this variable provides sensitivity to the type of mediation. The impact of realistic selection cuts on the $\cos \theta_{l l}$ distributions has then been studied, and we observed that $\cos \theta_{l l}$ looses discriminating power in the signal region $\mathrm{SR}_{t W}$, specifically designed to search for the $t W+E_{T}^{\text {miss }}$ signature. Since in $\mathrm{SR}_{t W}$ around half of the dilepton events are due to $t W+E_{T}^{\text {miss }}$ and the other half arises from $t \bar{t}+E_{T}^{\text {miss }}$, and given that the $\cos \theta_{l l}$ spectrum of both the $t \bar{t}+E_{T}^{\text {miss }}$ and the $t W+E_{T}^{\text {miss }}$ signals after the $\mathrm{SR}_{t W}$ selections is significantly different from the $\mathrm{SM}$ spectrum (see figure 7 ), a shape fit to the $\cos \theta_{l l}$ variable however still turns out to enhance the search sensitivity in practice.

After a brief description of the MC generation and our detector simulation, we have then presented our analysis strategy. Like in the earlier study [10], the observables $E_{T}^{\text {miss }}$ and $m_{T 2}$ provide the main handles to suppress the SM backgrounds in our work. In order to separate events that are associated to two $t \rightarrow b W \rightarrow b l \nu$ decays from those with one $t \rightarrow b W \rightarrow b l \nu$ decay and one $W \rightarrow l \nu$ decay, we cut on the $m_{b l}^{t}$ observable. With this selection we are able to split the search into two distinct signal regions (cf. figure 5). In the first signal region $\mathrm{SR}_{t \bar{t}}$ around $90 \%$ of the dilepton events arise from $t \bar{t}+E_{T}^{\text {miss }}$, while $10 \%$ are due to $t W+E_{T}^{\text {miss }}$. In contrast, in the second signal region $\mathrm{SR}_{t W}$ approximately $50 \%$ of the two-lepton events come from $t W+E_{T}^{\text {miss }}$ and the remaining $50 \%$ originate from $t \bar{t}+E_{T}^{\text {miss }}$ production.

We have then analysed the coverage of the parameter space of the spin-0 $s$-channel DM simplified models expected at LHC Run-3 and the HL-LHC. For mediator masses below about $150 \mathrm{GeV}$, we found that a search based on $\mathrm{SR}_{t \bar{t}}$ typically performs better than one using $\mathrm{SR}_{t W}$, while for heavier spin-0 states the opposite tends to be the case. Since the two signal regions $\mathrm{SR}_{t \bar{t}}$ and $\mathrm{SR}_{t W}$ do not overlap, they can be statistically combined, and it turns out that the combination yields 95\% CL exclusion bounds on the mediator masses that are improved significantly compared to previous projections [10, 11, 13, 14]. Numerically, we find for the DMF benchmark parameters $m_{\chi}=1 \mathrm{GeV}$ and $g_{\chi}=g_{t}=1$, the limits $M_{\phi, a} \gtrsim 410 \mathrm{GeV}$ and $M_{\phi, a} \gtrsim 530 \mathrm{GeV}$ at LHC Run-3 and the HL-LHC, respectively (see figure 8). It should be possible to further strengthen the quoted bounds by performing a shape fit to $C_{\mathrm{em}}$ in the signal regions $\mathrm{SR}_{t \bar{t}}$ and $\mathrm{SR}_{t W}$. The obtained improvements depend, however, strongly on the uncertainty on the shape of the $C_{\mathrm{em}}$ distribution within the SM. A reliable estimate of this uncertainty is only possible in an analysis that uses real LHC data, and in consequence we have not performed $C_{\mathrm{em}}$ shape fits in this article.

The analysis strategies proposed by us can also be applied to the production of DM in association with top quarks in the framework of next-generation DM simplified models such as for example the $2 \mathrm{HDM}+$ a model. A combined study of the $t \bar{t}+E_{T}^{\text {miss }}[19]$ and $t W+E_{T}^{\text {miss }}[15]$ signatures in the context of the $2 \mathrm{HDM}+$ a model, while beyond the scope of the work at hand, seems to be a worthwhile exercise.

\section{Acknowledgments}

We are grateful to Augustinas Malinauskas, Priscilla Pani, Deborah Pinna and Giulia Zanderighi for useful discussions. 
Open Access. This article is distributed under the terms of the Creative Commons Attribution License (CC-BY 4.0), which permits any use, distribution and reproduction in any medium, provided the original author(s) and source are credited.

\section{References}

[1] J. Abdallah et al., Simplified Models for Dark Matter Searches at the LHC, Phys. Dark Univ. 9-10 (2015) 8 [arXiv:1506.03116] [INSPIRE].

[2] D. Abercrombie et al., Dark Matter Benchmark Models for Early LHC Run-2 Searches: Report of the ATLAS/CMS Dark Matter Forum, arXiv:1507.00966 [INSPIRE].

[3] G. Busoni et al., Recommendations on presenting LHC searches for missing transverse energy signals using simplified s-channel models of dark matter, arXiv:1603.04156 [INSPIRE].

[4] G. D'Ambrosio, G.F. Giudice, G. Isidori and A. Strumia, Minimal flavor violation: An effective field theory approach, Nucl. Phys. B 645 (2002) 155 [hep-ph/0207036] [InSPIRE].

[5] M.R. Buckley, D. Feld and D. Goncalves, Scalar Simplified Models for Dark Matter, Phys. Rev. D 91 (2015) 015017 [arXiv: 1410.6497] [INSPIRE].

[6] U. Haisch and E. Re, Simplified dark matter top-quark interactions at the LHC, JHEP 06 (2015) 078 [arXiv : 1503.00691] [INSPIRE].

[7] M. Backović, M. Krämer, F. Maltoni, A. Martini, K. Mawatari and M. Pellen, Higher-order QCD predictions for dark matter production at the LHC in simplified models with s-channel mediators, Eur. Phys. J. C 75 (2015) 482 [arXiv: 1508.05327] [INSPIRE].

[8] M.R. Buckley and D. Goncalves, Constraining the Strength and CP Structure of Dark Production at the LHC: the Associated Top-Pair Channel, Phys. Rev. D 93 (2016) 034003 [arXiv: 1511.06451] [INSPIRE].

[9] C. Arina et al., A comprehensive approach to dark matter studies: exploration of simplified top-philic models, JHEP 11 (2016) 111 [arXiv:1605.09242] [INSPIRE].

[10] U. Haisch, P. Pani and G. Polesello, Determining the CP nature of spin-0 mediators in

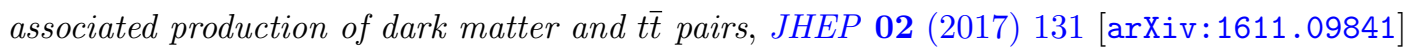
[INSPIRE].

[11] D. Pinna, A. Zucchetta, M.R. Buckley and F. Canelli, Single top quarks and dark matter, Phys. Rev. D 96 (2017) 035031 [arXiv:1701.05195] [INSPIRE].

[12] CMS collaboration, Search for dark matter produced in association with a single top quark or a top quark pair, CMS-PAS-EXO-18-010 [INSPIRE].

[13] ATLAS collaboration, ATLAS sensitivity to dark matter produced in association with heavy quarks at the HL-LHC, ATL-PHYS-PUB-2018-036 (2018).

[14] T. Plehn, J. Thompson and S. Westhoff, Dark Matter from Electroweak Single Top Production, Phys. Rev. D 98 (2018) 015012 [arXiv:1712.08065] [INSPIRE].

[15] P. Pani and G. Polesello, Dark matter production in association with a single top-quark at the LHC in a two-Higgs-doublet model with a pseudoscalar mediator, Phys. Dark Univ. 21 (2018) 8 [arXiv: 1712.03874] [INSPIRE].

[16] S. Ipek, D. McKeen and A.E. Nelson, A Renormalizable Model for the Galactic Center Gamma Ray Excess from Dark Matter Annihilation, Phys. Rev. D 90 (2014) 055021 [arXiv:1404.3716] [INSPIRE]. 
[17] J.M. No, Looking through the pseudoscalar portal into dark matter: Novel mono-Higgs and mono-Z signatures at the LHC, Phys. Rev. D 93 (2016) 031701 [arXiv:1509.01110] [INSPIRE].

[18] D. Goncalves, P.A.N. Machado and J.M. No, Simplified Models for Dark Matter Face their Consistent Completions, Phys. Rev. D 95 (2017) 055027 [arXiv: 1611.04593] [InSPIRE].

[19] M. Bauer, U. Haisch and F. Kahlhoefer, Simplified dark matter models with two Higgs doublets: I. Pseudoscalar mediators, JHEP 05 (2017) 138 [arXiv: 1701.07427] [INSPIRE].

[20] P. Tunney, J.M. No and M. Fairbairn, Probing the pseudoscalar portal to dark matter via $\bar{b} b Z(\rightarrow \ell \ell)+\mathbb{E}_{T}:$ From the LHC to the Galactic Center excess, Phys. Rev. D 96 (2017) 095020 [arXiv: 1705.09670] [INSPIRE].

[21] LHC Dark Matter Working Group collaboration, LHC Dark Matter Working Group: Next-generation spin-0 dark matter models, arXiv:1810.09420 [INSPIRE].

[22] N.F. Bell, G. Busoni and I.W. Sanderson, Self-consistent Dark Matter Simplified Models with an s-channel scalar mediator, JCAP 03 (2017) 015 [arXiv: 1612.03475] [INSPIRE].

[23] N.F. Bell, Y. Cai, J.B. Dent, R.K. Leane and T.J. Weiler, Dark matter at the LHC: Effective field theories and gauge invariance, Phys. Rev. D 92 (2015) 053008 [arXiv:1503.07874] [INSPIRE].

[24] F. Kahlhoefer, K. Schmidt-Hoberg, T. Schwetz and S. Vogl, Implications of unitarity and gauge invariance for simplified dark matter models, JHEP 02 (2016) 016 [arXiv: 1510.02110] [INSPIRE].

[25] N.F. Bell, Y. Cai and R.K. Leane, Mono-W Dark Matter Signals at the LHC: Simplified Model Analysis, JCAP 01 (2016) 051 [arXiv:1512.00476] [INSPIRE].

[26] U. Haisch, F. Kahlhoefer and T.M.P. Tait, On Mono-W Signatures in Spin-1 Simplified Models, Phys. Lett. B 760 (2016) 207 [arXiv: 1603.01267] [INSPIRE].

[27] C. Englert, M. McCullough and M. Spannowsky, S-Channel Dark Matter Simplified Models and Unitarity, Phys. Dark Univ. 14 (2016) 48 [arXiv:1604.07975] [InSPIRE].

[28] S. Dawson, The Effective W Approximation, Nucl. Phys. B 249 (1985) 42 [INSPIRE].

[29] G.L. Kane, W.W. Repko and W.B. Rolnick, The Effective $W^{ \pm}, Z^{0}$ Approximation for High-Energy Collisions, Phys. Lett. 148B (1984) 367 [INSPIRE].

[30] F. Maltoni, K. Paul, T. Stelzer and S. Willenbrock, Associated production of Higgs and single top at hadron colliders, Phys. Rev. D 64 (2001) 094023 [hep-ph/0106293] [INSPIRE].

[31] M. Farina, C. Grojean, F. Maltoni, E. Salvioni and A. Thamm, Lifting degeneracies in Higgs couplings using single top production in association with a Higgs boson, JHEP 05 (2013) 022 [arXiv: 1211.3736] [INSPIRE].

[32] S. Dawson and L. Reina, QCD corrections to associated Higgs boson production, Phys. Rev. D 57 (1998) 5851 [hep-ph/9712400] [INSPIRE].

[33] S. Dittmaier, M. Krämer, Y. Liao, M. Spira and P.M. Zerwas, Higgs radiation off quarks in supersymmetric theories at $e^{+} e^{-}$colliders, Phys. Lett. B 478 (2000) 247 [hep-ph/0002035] [INSPIRE].

[34] G. Bélanger, R.M. Godbole, C.K. Khosa and S.D. Rindani, Probing CP nature of a mediator in associated production of dark matter with single top quark, in 11th International Workshop on Top Quark Physics (TOP2018) Bad Neuenahr, Germany, September 16-21, 2018, arXiv: 1811.11048 [INSPIRE]. 
[35] J. Alwall et al., The automated computation of tree-level and next-to-leading order differential cross sections and their matching to parton shower simulations, JHEP 07 (2014) 079 [arXiv: 1405.0301] [INSPIRE].

[36] NNPDF collaboration, Parton distributions for the LHC Run II, JHEP 04 (2015) 040 [arXiv: 1410.8849] [INSPIRE].

[37] P. Artoisenet, R. Frederix, O. Mattelaer and R. Rietkerk, Automatic spin-entangled decays of heavy resonances in Monte Carlo simulations, JHEP 03 (2013) 015 [arXiv:1212.3460] [INSPIRE].

[38] T. Sjöstrand et al., An Introduction to PYTHIA 8.2, Comput. Phys. Commun. 191 (2015) 159 [arXiv: 1410.3012] [INSPIRE].

[39] F. Demartin, B. Maier, F. Maltoni, K. Mawatari and M. Zaro, tWH associated production at the LHC, Eur. Phys. J. C 77 (2017) 34 [arXiv:1607.05862] [InSPIRE].

[40] U. Haisch and G. Polesello, Searching for heavy Higgs bosons in the $t \bar{t} Z$ and $t b W$ final states, JHEP 09 (2018) 151 [arXiv:1807.07734] [INSPIRE].

[41] S. Frixione, E. Laenen, P. Motylinski, B.R. Webber and C.D. White, Single-top hadroproduction in association with a $W$ boson, JHEP 07 (2008) 029 [arXiv:0805.3067] [INSPIRE].

[42] ATLAS collaboration, Search for dark matter produced in association with bottom or top quarks in $\sqrt{s}=13 \mathrm{TeV}$ pp collisions with the ATLAS detector, Eur. Phys. J. C 78 (2018) 18 [arXiv: 1710.11412] [INSPIRE].

[43] J.M. Campbell, R.K. Ellis, P. Nason and E. Re, Top-Pair Production and Decay at NLO Matched with Parton Showers, JHEP 04 (2015) 114 [arXiv:1412.1828] [INSPIRE].

[44] E. Re, Single-top Wt-channel production matched with parton showers using the POWHEG method, Eur. Phys. J. C 71 (2011) 1547 [arXiv:1009.2450] [INSPIRE].

[45] T. Melia, P. Nason, R. Röntsch and G. Zanderighi, $W^{+} W^{-}, W Z$ and $Z Z$ production in the POWHEG BOX, JHEP 11 (2011) 078 [arXiv:1107.5051] [INSPIRE].

[46] P. Nason and G. Zanderighi, $W^{+} W^{-}, W Z$ and $Z Z$ production in the POWHEG-BOX-V2, Eur. Phys. J. C 74 (2014) 2702 [arXiv:1311.1365] [InSPIRE].

[47] S. Alioli, P. Nason, C. Oleari and E. Re, A general framework for implementing NLO calculations in shower Monte Carlo programs: the POWHEG BOX, JHEP 06 (2010) 043 [arXiv: 1002.2581] [INSPIRE].

[48] M. Czakon and A. Mitov, Top++: A Program for the Calculation of the Top-Pair Cross-Section at Hadron Colliders, Comput. Phys. Commun. 185 (2014) 2930 [arXiv:1112.5675] [INSPIRE].

[49] M. Czakon, P. Fiedler and A. Mitov, Total Top-Quark Pair-Production Cross Section at Hadron Colliders Through $\mathcal{O}\left(\alpha_{S}^{4}\right)$, Phys. Rev. Lett. 110 (2013) 252004 [arXiv:1303.6254] [INSPIRE].

[50] C. Anastasiou, L.J. Dixon, K. Melnikov and F. Petriello, High precision QCD at hadron colliders: Electroweak gauge boson rapidity distributions at NNLO, Phys. Rev. D 69 (2004) 094008 [hep-ph/0312266] [INSPIRE].

[51] R. Gavin, Y. Li, F. Petriello and S. Quackenbush, W Physics at the LHC with FEWZ 2.1, Comput. Phys. Commun. 184 (2013) 208 [arXiv:1201.5896] [InSPIRE].

[52] M. Cacciari, G.P. Salam and G. Soyez, FastJet User Manual, Eur. Phys. J. C 72 (2012) 1896 [arXiv: 1111.6097] [INSPIRE]. 
[53] M. Cacciari, G.P. Salam and G. Soyez, The anti- $k_{t}$ jet clustering algorithm, JHEP 04 (2008) 063 [arXiv: 0802.1189] [INSPIRE].

[54] ATLAS collaboration, The ATLAS Experiment at the CERN Large Hadron Collider, 2008 JINST 3 S08003 [INSPIRE].

[55] ATLAS collaboration,, Expected performance of the ATLAS experiment: detector, trigger and physics, CERN-OPEN-2008-020 [inSPIRE].

[56] C.G. Lester and D.J. Summers, Measuring masses of semiinvisibly decaying particles pair produced at hadron colliders, Phys. Lett. B 463 (1999) 99 [hep-ph/9906349] [INSPIRE].

[57] A. Barr, C. Lester and P. Stephens, m(T2): The truth behind the glamour, J. Phys. G 29 (2003) 2343 [hep-ph/0304226] [INSPIRE].

[58] ATLAS collaboration, Technical Design Report for the Phase-II Upgrade of the ATLAS TDAQ System, CERN-LHCC-2017-020 (2017).

[59] A.L. Read, Presentation of search results: The CL(s) technique, J. Phys. G 28 (2002) 2693 [INSPIRE].

[60] L. Moneta et al., The RooStats Project, PoS (ACAT2010) 057 (2010) [arXiv:1009.1003] [INSPIRE]. 